\title{
ORIGINAL
}

\section{MODELOS MATEMÁTICOS UTILIZADOS EN EL ESTUDIO DE LAS ENFERMEDADES TRANSMISIBLES}

\author{
Martí Casals (1, 2, 3), Katty Guzmán (1), Joan A. Caylà (1, 2). \\ (1) Servicio de Epidemiología, Agencia de Salud Pública de Barcelona. \\ (2) CIBER de Epidemiología y Salud Pública (CIBERESP). España. \\ (3) Departament de ciencies basiques. Universidad Internacional de Catalunya.
}

\section{RESUMEN}

Fundamento: Las enfermedades infecciosas han tenido históricamente un gran impacto en la morbilidad y en la mortalidad, lo que seguramente propició que desde hace siglos se hicieran predicciones sobre la evolución de las epidemias. El objetivo del trabajo es identificar los modelos matemáticos más utilizados y conocer las enfermedades en que más se aplicaron.

Métodos: Se revisaron las publicaciones indexadas en el sistema Medline entre el 1 de enero del 2000 y el 31 de agosto del 2009 y se procedió a una lectura de cada resumen, descartándose aquellos artículos que no se ajustaban al objetivo de este estudio. Se recogió el tipo de enfermedad infecciosa estudiada y de modelo matemático, la técnica estadística utilizada, el modelo de transmisión y el país del primer autor.

Resultados: Se seleccionó un total de 162 artículos de entre 617 posibles. La evolución de los artículos por años muestra una tendencia creciente a partir del año 2005. Por tipo de enfermedad, destacan las enfermedades infecciosas sin especificar, seguido de VIH-SIDA, malaria, tuberculosis, etc. Por modelos matemáticos hubo un predominio de los modelos estocásticos. Por país del primer autor, destacan los países europeos, sobretodo Gran Bretaña, y EEUU. El modelo de transmisión más utilizado ha sido el modelo SIR (21 casos/451). Del total de 58 artículos con una técnica estadística identificada se observó que en $12(20,7 \%)$ se utilizaban los modelos "Generalized Linear Model" y 11 (19.0\%) modelos de Markov.

Conclusiones: La modelización de las enfermedades transmisibles despierta un interés creciente y cabe esperar grandes innovaciones en los próximos años, sobretodo si su uso se extiende y se aplican a las enfermedades transmisibles "olvidadas" o a otros problemas de salud.

Palabras clave: Enfermedades transmisibles. Artículo de revista. Modelo matemático.

Correspondencia:

Martí Casals

Servicio de Epidemiología

Agència de Salut Pública de Barcelona

Pl Lesseps, 1

08023-Barcelona

Email: mcasals@aspb.cat

\section{ABSTRACT \\ Mathematical Models Used in the Study of Infectious Diseases}

Background: Infectious diseases have historically had a large impact on morbidity and mortality, which probably led predictions about the evolution of epidemics have been made for centuries. The objective is to identify the most-frequently used mathematical models and the diseases to which they are applied.

Methods: Publications indexed in Medline between 1 January 2000 and 31 August 2009 were reviewed: each abstract was read and articles that did not comply with the objectives of the study were discarded. The type of infectious disease, the mathematical model applied, the statistical technique used, the model of transmission and the country of the first author were collected.

Results: Of 617 possible articles, 162 were finally selected. The evolution of articles by years shows a rising trend since 2005. The most-common disease types were unespecified infectious diseases, HIV-AIDS, malaria and tuberculosis. Among mathematical models there was a predominance of stochastic models. The most-common country of the first author included the European countries, especially UK and USA. The most-widely used model of transmission was the SIR model (21 cases/451). Of the 58 articles which identified a statistical technique, 12 (20.7\%) used generalized linear models and 11 (19.0\%) used Markov models.

Conclusions: There is growing interest in the modelling of communicable diseases and substantial innovations may be expected in forthcoming years, above all if their use is extended and applied to "forgotten" communicable diseases or other health problems.

Keywords: Communicable diseases. Journal Article [Publication Type]. Mathematical model. 


\section{INTRODUCCIÓN}

Las enfermedades infecciosas han tenido históricamente un gran impacto en la morbilidad y en la mortalidad, lo que seguramente propició que desde hace siglos se hicieran predicciones sobre la evolución de las epidemias. El primer artículo identificado se debe a Bernouilli que desarrolló una fórmula en relación a la viruela que publicó en 1766, aunque 5 años antes D'Alambert había desarrollado un método que tenía en cuenta riesgos competitivos de muerte ${ }^{1}$. Otro precursor relevante fue Farr, quien en 1840 modeló la evolución de una enfermedad que tenía el ganado vacuno y que en nuestros días ha sido aplicada a la evolución del sida ${ }^{2}$.

Las áreas de la estadística que más han influenciado en la biomedicina en los últimos años han sido los modelos lineales generalizados (incluida la regresión lineal múltiple), análisis de supervivencia, análisis de datos categóricos, estadística espacial y los métodos bayesianos (en ensayos clíni$\cos$ y epidemiología). Los meta-análisis también han sido de gran interés en los últimos años ${ }^{3,4}$.

En 1994 sugirieron nuevos métodos estadísticos que tendrían un rol importante, como son el método "boostrap", los métodos bayesianos, los modelos generalizados aditivos, árboles de clasificación y regresión, modelos para datos longitudinales GEE ("general estimating equations"), modelos para datos jerárquicos y redes neuronales ${ }^{4,5}$.

Actualmente los nuevos avances tecnológicos en biomedicina hacen que la creación de equipos multidisciplinarios sean de vital importancia. Estos grupos pueden estar formados por clínicos, epidemiólogos, matemáticos, estadísticos, informáticos, biólogos, físicos, etc. En este sentido se ha publicado un estudio en el que se reivindica la importancia de la bioestadística en la investigación médica ${ }^{6}$.
El gran desarrollo adquirido por diferentes ciencias, como la epidemiología, las matemáticas, la estadística, la informática, ha propiciado que actualmente se hayan generado nuevas técnicas en procedimientos emergentes y recurrentes en el campo de la bioestadística y bioinformática (estadística espacial, redes neuronales y análisis de datos funcionales, etc) para describir la importancia de los modelos matemáticos en las enfermedades infecciosas.

El objetivo de este trabajo es identificar los modelos matemáticos más utilizados para predecir la evolución de las enfermedades transmisibles y las enfermedades en las que más frecuentemente se aplicaron.

\section{MATERIAL Y MÉTODOS}

Se revisaron las publicaciones indexadas en el sistema Medline entre el 1 de enero del 2000 y el 31 de agosto del 2009, utilizando las palabras clave: "Infectious disease and Stochastic model", "Infectious disease and Deterministic model ", "Mathematical and transmission and infectious disease", "Stochastic model and aids", "Deterministic model and aids", "Deterministic model and tuberculosis", "Stochastic model and tuberculosis", "Deterministic model and hepatitis", "Deterministic model and malaria", "Stochastic model and malaria", "Deterministic model meningococcal disease", "Stochastic model and meningococcal disease".

Se procedió a la lectura de cada resumen de los artículos detectados, descartándose aquéllos que no se ajustaban al objetivo de este estudio.

Se creó una base de datos que de cada artículo recogía las siguientes variables: Tipo de enfermedad infecciosa y de modelo matemático, técnica estadística utilizada, modelo de transmisión y país del primer autor. Los modelos matemáticos se clasificaron en dos grupos: estocásticos y deter- 
minísticos. En los estocásticos no es posible controlar los factores que intervienen en el estudio del fenómeno y, en consecuencia, no producen resultados únicos. En un modelo determinístico se pueden controlar los factores que intervienen en el estudio del proceso o fenómeno y por tanto se puede predecir con exactitud sus resultados. La diferencia más importante en el ámbito de la epidemiología radica en que en el modelo determinístico un solo sujeto causa una epidemia generalizada, mientras que bajo un modelo estocástico existe la posibilidad de que la epidemia se extinga ${ }^{7,8}$.

Los artículos se clasificaron también por los modelos de transmisión. En los modelos epidemiológicos se parte del supuesto de que los individuos de una población se dividen en tres grupos: individuos susceptibles (S) que son aquellos individuos que pueden contraer la enfermedad, individuos infectados (I) aquellos que poseen la enfermedad y son capaces de transmitirla, e individuos recuperados $(\mathrm{R})$ que son aquellos que se han recuperado de la enfermedad. Teniendo en cuenta la dinámica de los diferentes tipos de individuos, los principales modelos de transmisión de enfermedades infecciosas son: modelos
SIS, modelos SIR y modelos SEIR. Para la mayoría de las infecciones de transmisión sexual (ITS) resulta más útil el modelo SIS en el que el individuo no queda inmunizado tras sufrir la enfermedad. En los modelos SIR los individuos infectados se vuelven inmunes a la enfermedad una vez que dejan de estarlo y pasan al estado recuperado. En los modelos SEIR los individuos tienen un periodo de latencia durante el cual no tiene capacidad de transmitir la enfermedad y, por lo tanto, el individuo se dice que está expuesto?.

\section{RESULTADOS}

Se identificaron 617 artículos, descartándose 455 por no estar directamente relacionados con el objetivo. También se detectaron 10 artículos repetidos, 2 de ellos repetidos 3 veces. Finalmente, se seleccionó un total de 162 artículos relacionados con el objetivo de este estudio.

La evolución de los artículos por años muestra una tendencia creciente a partir del 2005. Debe tenerse en cuenta que el estudio se cerró el 31-8-2009, lo que explica la disminución observada en este año (figura 1).

Figura 1

Evolución del número anual de artículos publicados sobre modelización de enfermedades transmisibles entre enero de 2000 y agosto de 2009

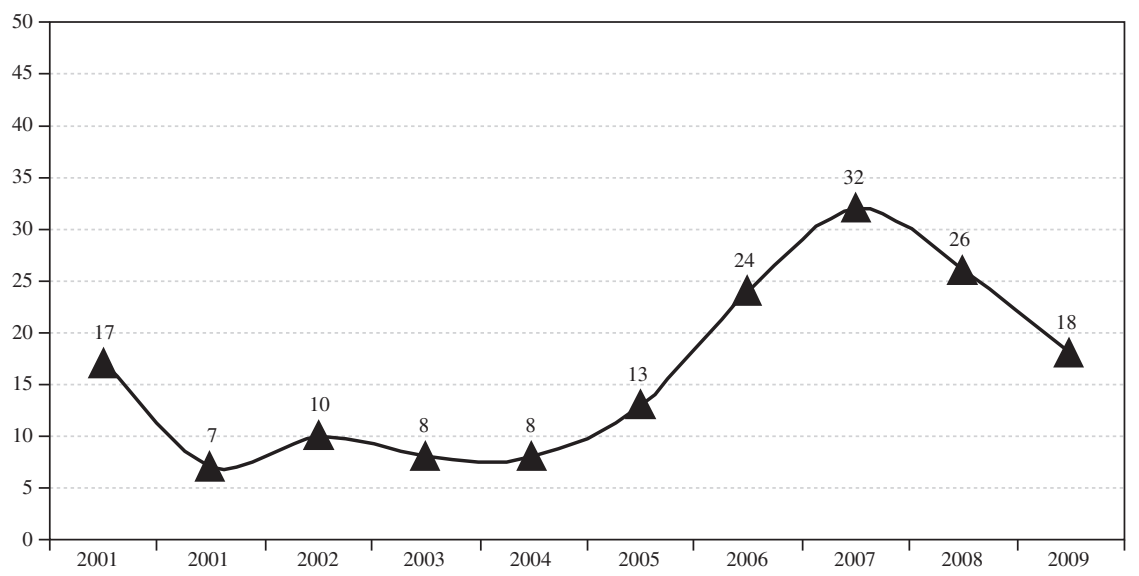


Figura 2

Evolución del número anual de artículos publicados sobre modelización de enfermedades transmisibles entre enero de 2000 y agosto de 2009 según tipo de enfermedad

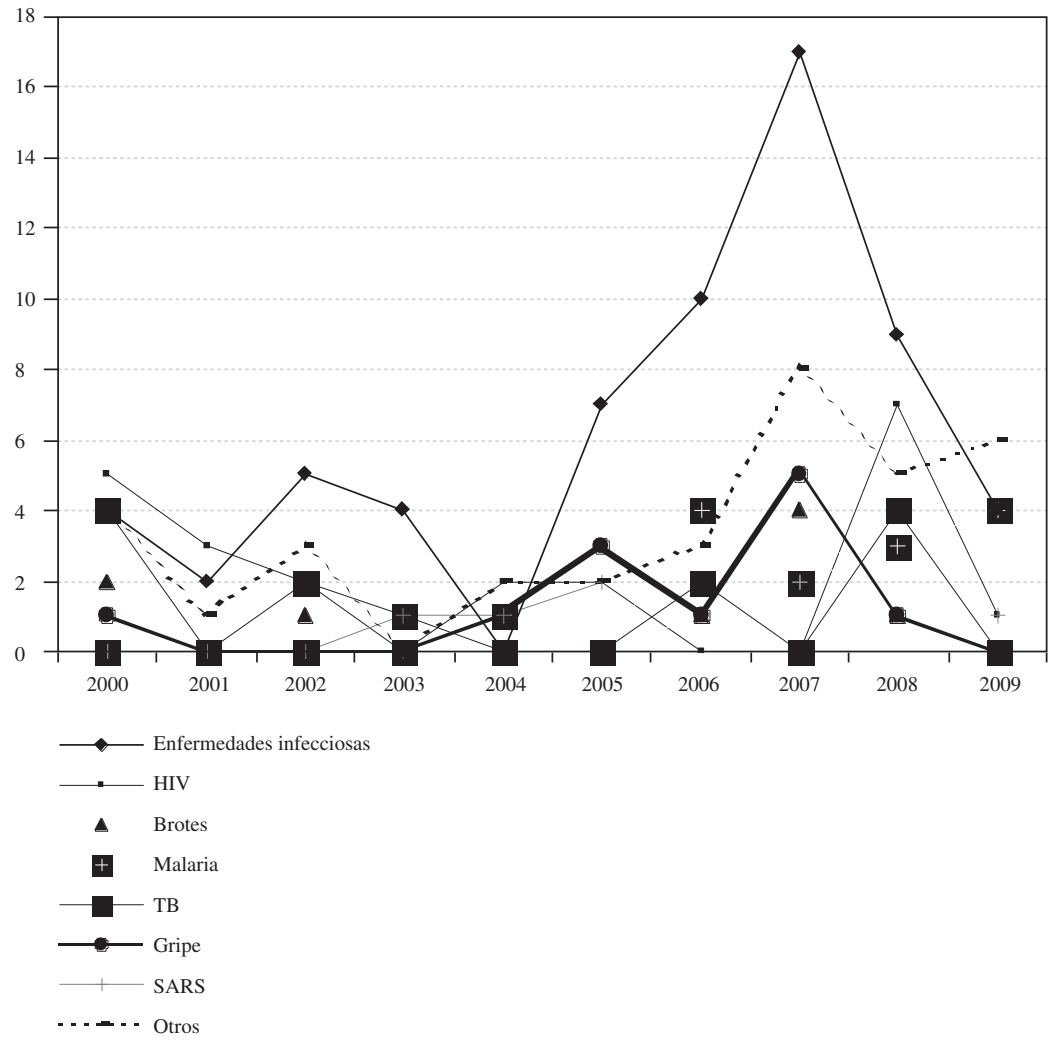

En relación al tipo de enfermedad infecciosa analizada destacan las enfermedades infecciosas sin especificar, seguidas de VIH-SIDA, malaria, tuberculosis, etc, mientras que para el SARS no aparecen publicaciones a partir de 2005 (figura 2).

La distribución de los artículos según el modelo matemático empleado muestra un predominio de los modelos estocásticos con unas tendencias a lo largo del estudio bastante paralelas entre ambos tipos de modelos (figura 3).

En cuanto a la distribución de los artículos por país del primer autor se aprecia un gran predominio de los países europeos, donde destaca Gran Bretaña, y de EEUU (figura 4). Se encontró tan sólo un artículo donde el país del primer autor era España.

El modelo de transmisión de enfermedades infecciosas más utilizado ha sido el modelo SIR (21 casos/ 45 total), seguido del modelo SIS (7casos/45 total) y otros (17/45 total) correspondían a los modelos SEIR, SI, SEIS o EIR.

Del total de 58 artículos con una técnica estadística identificada se observó que $12(20,7 \%)$ utilizaban los modelos 
Figura 3

Evolución del número anual de artículos publicados sobre modelización de enfermedades transmisibles entre enero de 2000 y agosto de 2009 según tipo de modelo matemático

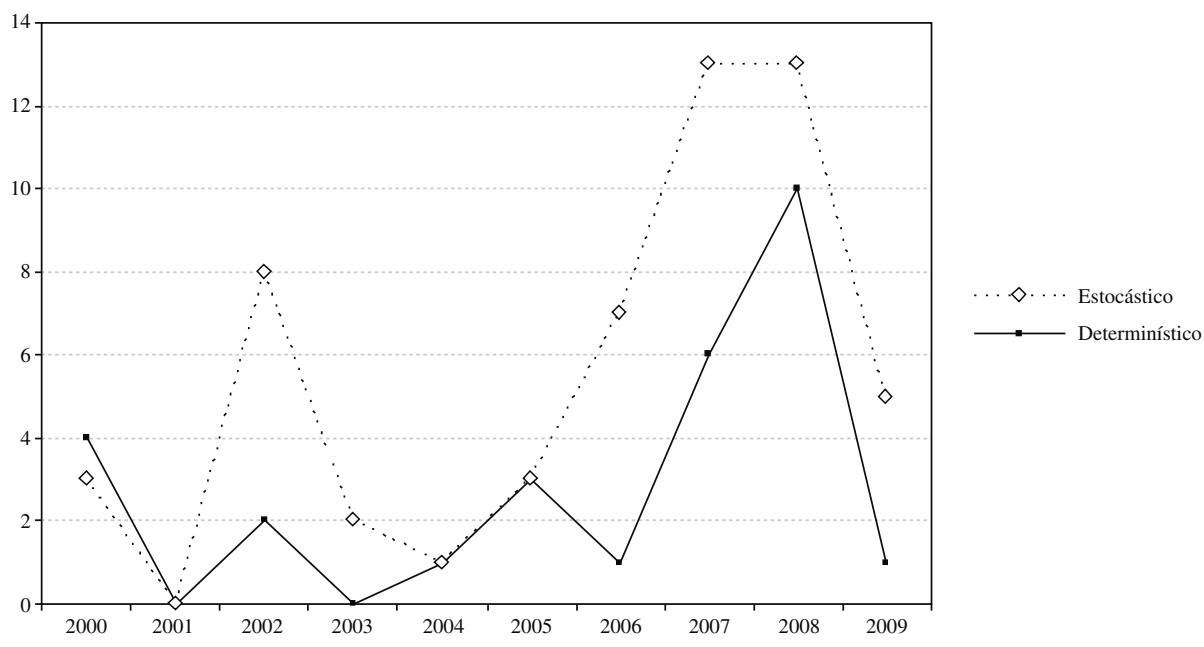

Figura 4

Evolución del número anual de artículos publicados sobre modelización de enfermedades transmisibles entre enero de 2000 y agosto de 2009 según país de los autores

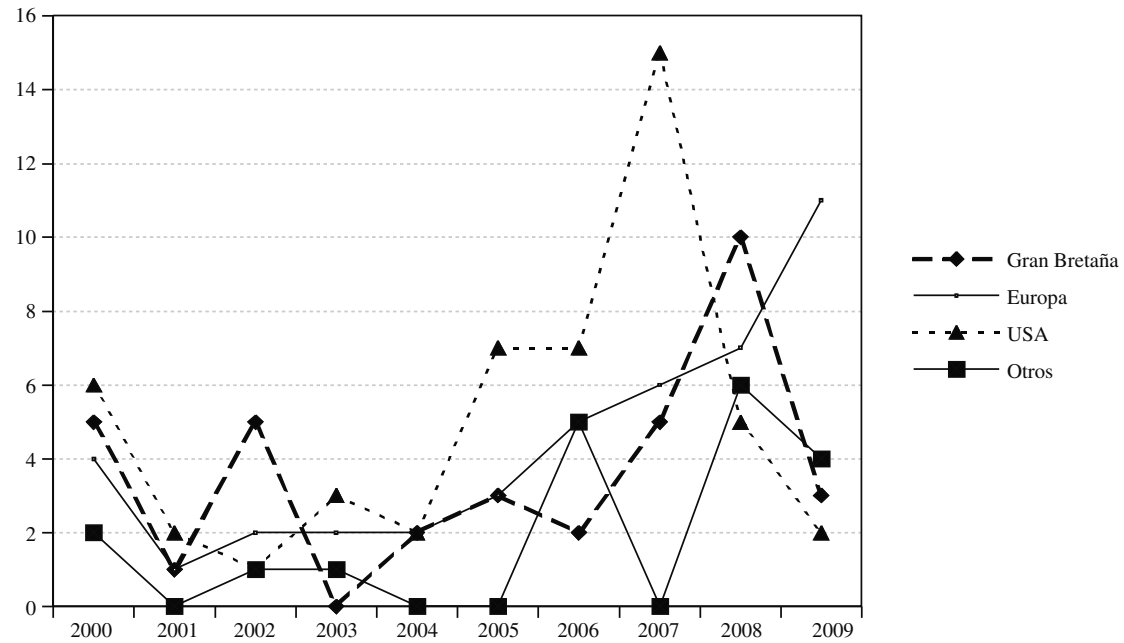

"Generalized Linear Model" (GLM), 11 $(19,0 \%)$ modelos de Markov, 9 (15,5\%) modelos espaciales, $9(15,5 \%)$ modelos bayesianos, 8 (13,8\%) modelos de Mon- tecarlo y $9(15,5 \%)$ otras técnicas (series temporales, redes neuronales o "Generalized Estimating Equations" (GEE)). 


\section{DISCUSIÓN}

En esta investigación se objetiva una tendencia creciente en la aplicación de los modelos matemáticos a lo largo del período de estudio. Analizando el tipo de enfermedades investigadas se aprecia también un incremento en los últimos años para el estudio de las enfermedades infecciosas sin especificar, pero también de HIVSIDA, malaria, tuberculosis y "otras", lo que seguramente refleja que estas técnicas son cada vez más populares y se están extendiendo a enfermedades en las que hasta ahora no se habían utilizados modelos. Justamente esta idea de probar estos modelos en enfermedades "olvidadas" ha permitido que en este número monográfico de la revista se presente también un artículo que recoge un modelo predictivo de la tuberculosis en Barcelona basado en series temporales.

Los modelos matemáticos en las enfermedades infecciosas con la finalidad de realizar predicciones constituye un desafío enorme desde hace siglos como se comenta en la introducción y que continúa en el momento actual. En las numerosas reuniones científicas que se suceden actualmente en estos días en relación a la gripe A/H1N1 es motivo de gran discusión si la tasa de ataque será del $30 \%$ o del $10 \%$, por ejemplo, o cuál será el porcentaje de personas hospitalizadas o su letalidad. Ya se han desarrollado aplicaciones informáticas como "Flu Surge" (creada la primera versión en el año 2004) de los CDC de EEUU, y ya se están publicando los primeros trabajos sobre esta materia, algunos muy sofisticados ${ }^{10}$.

Es interesante comentar la evolución del uso de modelos matemáticos en el SARS, detectado en el 2003 y que fue eliminado muy pronto. Las primeras publicaciones son del 2003 y a partir de 2005 ya no hay mas publicaciones sobre este tema, lo que denota que este tipo de investigaciones se desarrollan rápidamente debido al posible potencial pandémico y también se publican muy pronto debido al interés inusitado que despiertan.

En relación al predominio de los modelos estocásticos, probablemente se explicaría por la frecuencia de nuevas epidemias que hace que no se conozca el comportamiento de sus parámetros $\mathrm{y}$, por lo tanto, no se pueden presuponer los parámetros conocidos o 'determinados' en los modelos determinísticos 9 .

En cuanto al país del primer autor llama poderosamente la atención que casi todos los artículos proceden de EEUU y Europa, sobretodo de Gran Bretaña, con una escasa aportación española. En nuestro caso hemos podido apreciar la utilidad de estos modelos en temas como la enfermedad meningocócica ${ }^{11}$ y en la gripe estacional. Para esta última enfermedad nos basamos en la serie de entierros observados durante años en la ciudad de Barcelona y este mismo modelo ha sido útil en las muertes por olas de calor ${ }^{12}$. Por tanto sería de gran interés procurar que estos modelos estuvieran también al servicio de la vigilancia epidemiológica de todas nuestras CCAA y de muchos otros países.

Para diseñar los modelos de predicción apropiados para enfermedades infecciosas se ha utilizado en los últimos años modelos generales, como los modelos SIR basados en el método Montecarlo, modelos de cadenas de Markov, y modelos específicos para situaciones concretas (gaussianos para transmisión aerógena, meteorológicos, matrices de cálculo de datos retrospectivos,...). Los modelos GLM (inferencia frecuentista) eran anteriormente muy utilizados, pero en los últimos años es de más interés la utilización de modelos espaciales aplicando la inferencia bayesiana.

Entre las posibles limitaciones de este estudio debe citarse que es probable que 
haya artículos no detectados por el sistema de búsqueda realizado y que muchos de los artículos descartados lo fueron tras la lectura de los resúmenes. No obstante se estima que la investigación realizada es válida para estudiar las tendencias de la utilización de modelos matemáticos en las enfermedades transmisibles, y también para conocer las enfermedades que con más frecuencia los utilizan, en qué países se realizan este tipo de estudios y qué técnicas estadísticas se utilizan.

En conclusión, los modelos matemáticos en el estudio de las enfermedades transmisibles despierta un interés creciente que aumentará todavía más con los estudios que se están realizando en torno a la nueva gripe A/H1N1, o a posibles nuevas pandemias que sin duda se registraran en los próximos años. También cabe esperar innovaciones en los modelos matemáticos y técnicas estadísticas en la medida en que se apliquen a las enfermedades transmisibles "olvidadas" o a otros problemas de salud.

\section{BIBLIOGRAFÍA}

1. Dietz K, Heesterbeek JA. Daniel Bernoulli's epidemiological model revisited. Math Biosci. 2002; 180: 1-21.

2. Bregman DJ, Langmuir AD. Farr's law apllied to AIDS projections. JAMA. 1990; 263 (11): 1522-5.

3. Armitage P, Berry G. Statistical Methods in Medical Research (3rd edition). Blackwell; 1994.
4. Cadarso-Suárez C, González-Manteiga W. Statistics in biomedical research. Arbor. 2007. CLXXXIII (725): 353-361 .

5. Altman, D. G. and Goodman, s (1994): Transfer of technology from statistical journals to the biomedical literature: past trends and future predictions. J Am Med Assoc. 272-129.

6. Cobo E, Selva-O'Callagham A, Ribera JM, Cardellach F, Domínguez R, Vilardell M. Statistical reviewers improve reporting in biomedical articles: a randomized trial. PLoS ONE. 2007; 2: e332.

7. Brauer F, et al, Mathematical epidemiology. Mathematical biosciences subseries 1945. Berlin: Springer; 2008. p. 408.

8. Munayco EC. Conceptos fundamentales del modelamiento epidémico. Bol Epidemiol (Lima). 2009; 18(27): 528-9.

9. A. Martín del Rey. Epidemiología Matemática usando autómatas celulares sobre grafos. Actas del CEDYA 2009, Papel 35, 2009. Disponible en: http://matematicas.uclm.es/cedya09/archive/textos/35_Martin-del-Rey-A.pdf

10. Balcan D, Hu H, Goncalves B, Bajardi P, Poletto C, Ramasco JJ, et al. Seasonal transmission potential and activity peaks of the new influenza A (H1N1): a Monte Carlo likelihood analysis based on human mobility. BMC Med. 2009; 7: 45-50.

11. Dominguez A, Munoz P, Cardenosa, N, Martinez A, Cayla J; Meningococcal Disease Study Group. Time-series analysis of meningococcal disease in Catalonia. Ann of Epidemiol. 2007; 17 (9): 654662 .

12. Tobías A, de Olalla PG, Linares C, Bleda MJ, Caylà JA, Díaz J. Short-term effects of extreme hot summer temperatures on total daily mortality in Barcelona, Spain. Int J Biometeorol. 2009 Sep 24. 
